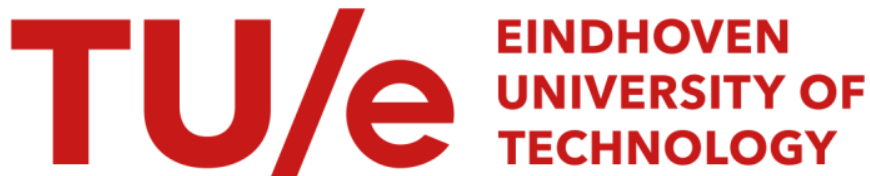

\section{Lyapunov-stability of solution branches of rotating disk flow}

\section{Citation for published version (APA):}

Eeten, van, K. M. P., Schaaf, van der, J., Heijst, van, G. J. F., \& Schouten, J. C. (2013). Lyapunov-stability of solution branches of rotating disk flow. Physics of Fluids, 25(7), 073602-1/13. [073602].

https://doi.org/10.1063/1.4812704

DOI:

$10.1063 / 1.4812704$

Document status and date:

Published: 01/01/2013

\section{Document Version:}

Publisher's PDF, also known as Version of Record (includes final page, issue and volume numbers)

\section{Please check the document version of this publication:}

- A submitted manuscript is the version of the article upon submission and before peer-review. There can be important differences between the submitted version and the official published version of record. People interested in the research are advised to contact the author for the final version of the publication, or visit the $\mathrm{DOI}$ to the publisher's website.

- The final author version and the galley proof are versions of the publication after peer review.

- The final published version features the final layout of the paper including the volume, issue and page numbers.

Link to publication

\section{General rights}

Copyright and moral rights for the publications made accessible in the public portal are retained by the authors and/or other copyright owners and it is a condition of accessing publications that users recognise and abide by the legal requirements associated with these rights.

- Users may download and print one copy of any publication from the public portal for the purpose of private study or research.

- You may not further distribute the material or use it for any profit-making activity or commercial gain

- You may freely distribute the URL identifying the publication in the public portal.

If the publication is distributed under the terms of Article 25fa of the Dutch Copyright Act, indicated by the "Taverne" license above, please follow below link for the End User Agreement:

www.tue.nl/taverne

Take down policy

If you believe that this document breaches copyright please contact us at:

openaccess@tue.nl

providing details and we will investigate your claim. 


\title{
Lyapunov-stability of solution branches of rotating disk flow
}

\author{
K. M. P. van Eeten, ${ }^{1}$ J. van der Schaaf, ${ }^{1}$ G. J. F. van Heijst, ${ }^{2}$ \\ and J. C. Schouten ${ }^{1, a)}$ \\ ${ }^{1}$ Laboratory of Chemical Reactor Engineering, Department of Chemical Engineering \\ and Chemistry, Eindhoven University of Technology, P.O. Box 513, 5600 MB Eindhoven, \\ The Netherlands \\ ${ }^{2}$ Fluid Dynamics Laboratory, Department of Applied Physics, Eindhoven University of \\ Technology, P.O. Box 513, 5600 MB Eindhoven, The Netherlands
}

(Received 7 March 2013; accepted 16 May 2013; published online 9 July 2013)

This paper investigates the stability of solutions to the problem of viscous flow between an infinite rotating disk and an infinite stationary disk. A random perturbation, satisfying the Von Kármán similarity transformation, is applied to the steady velocity profiles for four solution branches, after which the disturbance propagation is tracked as a function of time. It was found that three of the four solution branches (including the Batchelor solution) were Lyapunov-stable and the development of the Lyapunov-coefficients as a function of the Reynolds number was determined. Stewartson-type of flow was found to be unstable and developed into a flow field corresponding to the Batchelor-solution. The mechanism with which the non-viscous core in this latter type of flow acquired its angular momentum was identified as being dominated by radial convection towards the axis of rotation. (C) 2013 AIP Publishing LLC. [http://dx.doi.org/10.1063/1.4812704]

\section{INTRODUCTION}

The topic of laminar flow near rotating disks allows for a solution of the full Navier-Stokes equations and has hence received a lot of research attention starting with the pioneering work by Von Kármán in 1921. ${ }^{1}$ Von Kármán argued that the flow of an infinite fluid near an infinite rotating disk can be fully described by assuming that the fluid velocity perpendicular to the disk is a function of the distance to the disk only. Under this hypothesis, the fluid flow can be described by a class of so-called self-similar solutions. Von Kármán found that the velocity gradients were confined in a boundary layer attached to the disk, which the present authors would like to term a "Von Kármán boundary layer." " In this boundary layer, the azimuthal velocity gradually decreases from the azimuthal velocity of the disk to zero, while moving away from the disk. Also, in this boundary layer, fluid moves radially outwards due to the centrifugal action of the disk, which is balanced via continuity by an axial flow towards the disk.

Von Kármán's similarity transformation was later used by Bödewadt for the case of an infinitely extended rotating fluid over an infinite stationary disk. ${ }^{3}$ Similarly, it was found that a boundary layer is attached to the stationary disk. In this so-called Bödewadt boundary layer, the azimuthal velocity of the fluid gradually decreases from the azimuthal velocity of the fluid at infinity to zero, while approaching the disk. Due to the rotational motion of the fluid, the pressure in this boundary layer decreases towards the axis of rotation, invoking a radial inflow in this boundary layer. This centripetal flow is again balanced via continuity by an axial flow away from the disk.

The similarity hypothesis was later adopted by Batchelor to describe the flow between two infinite disks; one rotating and one stationary. ${ }^{4}$ Batchelor argued that the flow field would show

\footnotetext{
a) Author to whom correspondence should be addressed. Electronic mail: J.C.Schouten@tue.nl
} 
similarities to both Von Kármán's solution for the case where an infinite fluid was rotating over an infinite stationary disk, and the Bödewadt-solution where an infinite fluid rotates over an infinite stationary disk. According to Batchelor, a Bödewadt boundary layer is attached to the stationary disk, which is separated from a Von Kármán boundary layer at the rotating disk by a bulk region in solid-body rotation, with a uniform azimuthal velocity and zero radial velocity.

Batchelor's views were completely opposed by Stewartson, who argued on the basis of the same similarity transformation that apart from a Von Kármán layer near the rotating disk, the main body of the fluid would be at rest. ${ }^{5}$ The existence and uniqueness of either of these solutions was intensely debated over the following decades until in 1977 a numerical study by Holodniok et $a .^{6}$ revealed that there is not a unique solution to the system of equations derived via the similarity transformation. In fact, a whole multitude of solution branches was found, some of which seeming quite exotic or physically unrealistic. ${ }^{7}$

The question remains, however, how the different solution branches predicted by Holodniok et al., ${ }^{7}$ might be achieved from an arbitrary initial condition of the fluid and whether these solution branches are stable under a minor perturbation. This paper addresses these issues, all under the Von Kármán similarity hypothesis. It should be noted that an experimental procedure of adding a self-similar perturbation to a steady flow field which does not disturb the self-similar nature of the flow, might not be physically feasible. The present investigation will, however, shed some light on the stability of these hypothetical self-similar solutions, which are nonetheless still found to describe the interior flow field between finite sized disks. In fact, for Reynolds numbers up to the order $O\left(10^{3}\right)$ and radii up to $80 \%$ of the radius of the disks, Lopez et al. showed that the flow field can correctly be described with self-similar solutions. ${ }^{8}$ Also, any discrepancies in the observed stability of flow fields between finite sized disks and the stability of self-similar solutions can be discussed with regard to the boundary conditions at the edges of the disks.

The paper is structured as follows: first, the physical system is stated together with the governing equations describing the time-dependent flow field of a fluid between two infinite disks, one stationary and one rotating. Subsequently, the mathematical methods are described with which these equations are solved to yield flow fields corresponding to several solution branches. Then, in Sec. IV, the tools for analyzing the stability of the various solution branches are presented. Next, the results are presented for the development of the flow field, depending on different initial conditions, and its stability towards a minor disturbance. The paper finishes with some concluding remarks and a discussion on the effect of radially finite disks, with regard to the boundary condition at the edge of the disk. Also, the physical plausibility of self-similar perturbations which do not disturb the self-similar nature of the flow field is discussed in Sec. VI.

\section{PHYSICAL SYSTEM AND GOVERNING EQUATIONS}

Figure 1 shows a schematic drawing of the flow geometry, consisting of a rotating disk and a stationary disk with a disk spacing $h$. The flow is conveniently described in terms of a cylindrical coordinate system $(\hat{r}, \theta, \hat{z})$, being the radial, azimuthal, and axial coordinate, respectively. The

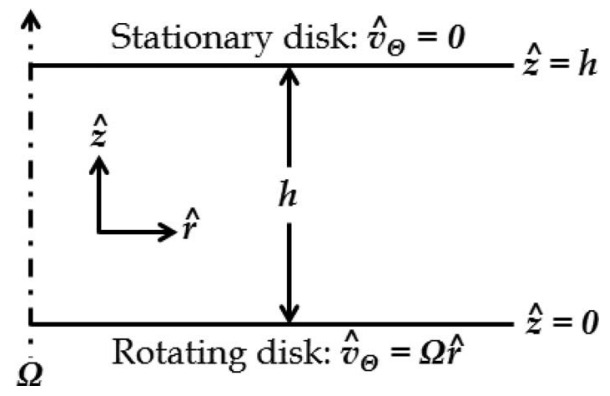

FIG. 1. Schematic drawing of the flow geometry, consisting of two parallel, infinite disks. The lower disk rotates with an angular velocity $\Omega$, while the upper disk is stationary. The distance between the disks is $h$. 
position $\hat{r}=0$ coincides with the rotation axis, the plane $\hat{z}=0$ represents the bottom disk, and the plane $\hat{z}=h$ corresponds to the top disk. The fluid flow is then described by the velocity vector $\underline{\hat{v}}=\left(\hat{v}_{r}, \hat{v}_{\theta}, \hat{v}_{z}\right)$ and depends on both position and time. The fluid is taken to be Newtonian with a density $\rho$ and a kinematic viscosity $\nu$. The pressure is a function of position and time and is denoted by $\hat{p}$. The angular velocity of both disks can be varied so that at some time before $\hat{t}=0$, a steady flow field of the fluid is obtained, which is given by $\underline{\hat{v}}_{0}$. Then, at $\hat{t}=0$ the top disk is impulsively put to rest, while the bottom disk is impulsively set to rotate at an angular velocity of $\Omega$ [rad s $\left.{ }^{-1}\right]$. All physical quantities are non-dimensionalized as follows:

$$
\begin{gathered}
t \equiv \hat{t} \Omega, \quad z \equiv \hat{z} h^{-1}, \quad r \equiv \hat{r} h^{-1}, \quad p \equiv \hat{p} \rho^{-1} \Omega^{-2} h^{-2} \\
\underline{v}=\left(v_{r}, v_{\theta}, v_{z}\right) \equiv\left(\hat{v}_{r}, \hat{v}_{\theta}, \hat{v}_{z}\right) \Omega^{-1} h^{-1}=\underline{\hat{v}} \Omega^{-1} h^{-1}
\end{gathered}
$$

For the case of rotational symmetry and incompressible flow, the three components of the Navier-Stokes equation in their dimensionless form can be written as

$$
\begin{gathered}
\frac{\partial v_{r}}{\partial t}+v_{r} \frac{\partial v_{r}}{\partial r}+v_{z} \frac{\partial v_{r}}{\partial z}-\frac{v_{\theta}^{2}}{r}=-\frac{\partial p}{\partial r}+\frac{1}{R e_{h}}\left(\frac{\partial^{2} v_{r}}{\partial z^{2}}+\frac{\partial^{2} v_{r}}{\partial r^{2}}+\frac{1}{r} \frac{\partial v_{r}}{\partial r}-\frac{v_{r}}{r^{2}}\right), \\
\frac{\partial v_{\theta}}{\partial t}+v_{r} \frac{\partial v_{\theta}}{\partial r}+v_{z} \frac{\partial v_{\theta}}{\partial z}+\frac{v_{r} v_{\theta}}{r}=\frac{1}{R e_{h}}\left(\frac{\partial^{2} v_{\theta}}{\partial z^{2}}+\frac{\partial^{2} v_{\theta}}{\partial r^{2}}+\frac{1}{r} \frac{\partial v_{\theta}}{\partial r}-\frac{v_{\theta}}{r^{2}}\right), \\
\frac{\partial v_{z}}{\partial t}+v_{r} \frac{\partial v_{z}}{\partial r}+v_{z} \frac{\partial v_{z}}{\partial z}=-\frac{\partial p}{\partial z}+\frac{1}{R e_{h}}\left(\frac{\partial^{2} v_{z}}{\partial z^{2}}+\frac{\partial^{2} v_{z}}{\partial r^{2}}+\frac{1}{r} \frac{\partial v_{z}}{\partial r}\right),
\end{gathered}
$$

while the continuity equation for incompressible flow takes the form

$$
\frac{\partial v_{r}}{\partial r}+\frac{v_{r}}{r}+\frac{\partial v_{z}}{\partial z}=0
$$

The Reynolds number of the flow is defined as

$$
R e_{h} \equiv \Omega h^{2} v^{-1}
$$

The initial condition of the fluid at $t=0$ is taken as a state of solid-body rotation, of which the azimuthal velocity can freely be chosen: $\underline{v}_{0}(r, z)=(0, s r, 0)$, in which $-1 \leq s \leq 1$. For $t>0$ the flow field has to satisfy the no-slip conditions at both disks. The boundary condition at the rotating disk for $t>0$ is given by

$$
\underline{v}_{z=0}=(0, r, 0),
$$

while at the stationary disk, for $t>0$, the flow has to satisfy

$$
\underline{v}_{z=1}=(0,0,0) \text {. }
$$

\section{METHODS OF SOLUTION}

For the case of steady, self-similar flow over an infinite rotating disk in an infinite fluid, Von Kármán argued that $v_{z}=v_{z}(z)$ is a function of $z$ only. ${ }^{1}$ For the present configuration, in which derivatives in time are allowed, a similar transformation is adopted, according to which $v_{z}=-2 H(z, t)$ is a function of both $z$ and $t$, allowing for changes in time as well as in space. It should be noted that in this way it is possible to calculate time-dependent solutions of the flow field, while still conforming to the Von Kármán similarity hypothesis. It can then be derived that

$$
v_{r}=r \frac{\partial H(z, t)}{\partial z},
$$




$$
\begin{gathered}
v_{\theta}=r G(z, t), \\
v_{z}=-2 H(z, t) .
\end{gathered}
$$

With these transformations, the equation of continuity (6) is automatically satisfied and the only relevant equations in the description of the flow field are the radial and azimuthal components of the Navier-Stokes equation. The $r$ and $\theta$-components of the Navier-Stokes equation (3) and (4) can then be rewritten in the form of two partial differential equations

$$
\begin{gathered}
\frac{\partial^{3} H}{\partial z^{2} \partial t}=R e_{h}^{-1} \frac{\partial^{4} H}{\partial z^{4}}+2\left(H \frac{\partial^{3} H}{\partial z^{3}}+G \frac{\partial G}{\partial z}\right), \\
\frac{\partial G}{\partial t}=R e_{h}^{-1} \frac{\partial^{2} G}{\partial z^{2}}+2\left(H \frac{\partial G}{\partial z}-G \frac{\partial H}{\partial z}\right) .
\end{gathered}
$$

For $t>0$, these equations have to be solved subject to the boundary conditions (8) and (9) at the rotating and non-rotating disk, respectively. These boundary conditions are rewritten in terms of $G(z, t)$ and $H(z, t)$ so that they take the following form:

$$
\begin{aligned}
& H(z=0, t)=0,\left.\quad \frac{\partial H}{\partial z}\right|_{(z=0, t)}=0, \quad G(z=0, t)=1, \\
& H(z=1, t)=0,\left.\quad \frac{\partial H}{\partial z}\right|_{(z=1, t)}=0, \quad G(z=1, t)=0 .
\end{aligned}
$$

The initial condition of the fluid velocity will be chosen so that the fluid is in solid-body rotation with angular velocity $s \Omega$, in which $-1 \leq s \leq 1$. The fluid at $t<0$ can thus be rotating in line with the rotational direction of the bottom disk (i.e., $s>0$ ), opposed to the rotational direction of the bottom disk (i.e., $s<0$ ), or the fluid can be initially at rest (i.e., $s=0$ ). The initial condition of the fluid is hence given by

$$
H(z, t=0)=0,\left.\quad \frac{\partial H}{\partial z}\right|_{(z, t=0)}=0, \quad G(z, t=0)=s .
$$

The system of partial differential equations (13) and (14), subject to the boundary conditions (15) and (16), and the initial condition (17) is solved numerically using a finite difference method. The axial coordinate between the disks is discretized in $n_{z}$ equidistant gridpoints $z_{i}(i=1,2,3, \ldots$, $n_{z}$ ), with $z_{1}=0$ and $z_{n_{z}}=1$. Time is discretized in $n_{t}$ equidistant gridpoints $t_{j}\left(j=1,2,3, \ldots, n_{t}\right)$, with $t_{1}=0$ and $t_{n_{t}}=t_{f}$. With this discretization, $G\left(z_{i}, t_{j}\right)$ and $H\left(z_{i}, t_{j}\right)$ are represented by $G_{i}^{j}$ and $H_{i}^{j}$. Their derivatives with respect to $z$ are approximated with a second order central finite difference to improve accuracy, whereas their derivatives with respect to $t$ are approximated with a first order backwards finite difference to enhance numerical stability by solving the system of finite difference equations implicitly. This will thus result in second order accuracy in $z$, so that the numerical error is of the order $O\left(n_{z}^{-2}\right)$, while the time-stepping error is of the order $O\left(t_{f} n_{t}^{-1}\right)$.

To determine the required resolution of the solution grid, an initial set of two simulations was run. In these numerical simulations, fluid with zero initial momentum was accelerated by the rotating disk at $z=0$ for the case $R e_{h}=1000$. For the first run, the number of grid points was taken to be $n_{z}=200$, while in the second run $n_{z}=100$. Since no qualitative difference between the two runs could be observed, for the remainder of the simulations, the number of grid points in $z$ was fixed to $n_{z}=100$, so that $z_{1}=0$ and $z_{100}=1$. The implicit method always resulted in converging solutions, regardless of the number of time steps that was chosen. The number of time steps was thus fixed at $n_{t}=200$, while the final value of the calculated time, $t_{f}$, was chosen so that

$$
\sum_{i=1}^{n_{z}}\left(G_{i}^{n_{t}}-G_{i}^{n_{t}-1}\right)^{2}<\epsilon,
$$


in which $\epsilon$ is the machine accuracy: $\epsilon=2^{-53} \approx 2.204 \times 10^{-16}$. A steady solution $\left(G^{\infty}(z), H^{\infty}(z)\right)$ is thus defined as a solution for which inequality (18) is first satisfied. This steady solution will then be tested for stability.

\section{STABILITY ANALYSIS}

A random perturbation was applied to a steady solution, after which the temporal evolution of this disturbance was numerically investigated. At $t=0$ a pseudo-random disturbance is added to $G_{i}^{\infty}$ so that

$$
G_{i}^{0}=G_{i}^{\infty}+\delta_{i}
$$

in which $\delta_{i}$ is a vector containing $n_{z}$ pseudo-random numbers between -0.005 and 0.005 . The pseudo-random numbers are generated with the 32-bit Mersenne Twister Algorithm and scaled to the interval $[-0.005,0.005]$. An exaggerated example of a disturbed azimuthal velocity profile is shown in Figure 2.

The disturbance propagation expressed in non-discretized form is defined as

$$
\Delta(t)=\int_{0}^{1}\left(G(z, t)-G^{\infty}(z)\right)^{2} d z
$$

and is numerically approximated using the trapezoid rule for numerical integration.

In theory, three situations may arise with respect to the stability of a steady solution branch, which are reflected in the limit as $t \rightarrow \infty$ of the function $\Delta(t)$ :

1. if $\lim _{t \rightarrow \infty} \Delta(t)=0$, the solution is stable and converges back to its original state;

2. if $\lim _{t \rightarrow \infty} \Delta(t)=\alpha$ and $\alpha$ is a (positive, real) constant, the solution is unstable and diverges from its original state to a new steady state;

3. if $\lim _{t \rightarrow \infty} \Delta(t)$ does not exist, the solution is unstable and diverges from its original state and never reaches any other steady state, i.e., either a periodic solution is obtained, or the system becomes chaotic.

For the first case, in which $\Delta(t) \rightarrow 0$ as $t \rightarrow \infty$, an additional analysis will be performed to check whether the solution is Lyapunov stable; i.e., does the disturbance to the steady velocity profile

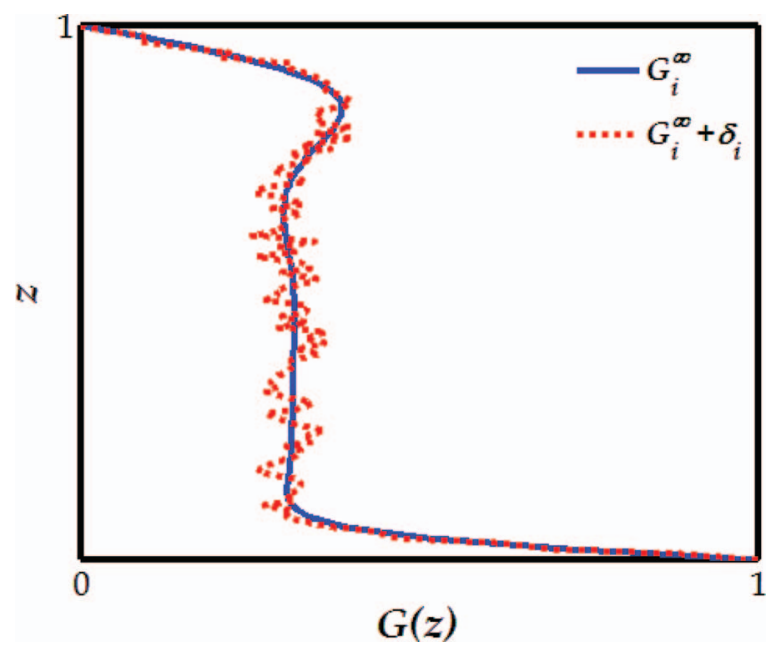

FIG. 2. A random disturbance $\delta_{i}$ is added to the azimuthal velocity profile $G(z)$. In this example figure, the order of the disturbance is exaggerated. The vector $\delta_{i}$ normally has $n_{z}$ random numbers between -0.005 and 0.005 . However, for visualization reasons, in this figure $\delta_{i}$ ranges from -0.05 to 0.05 . 


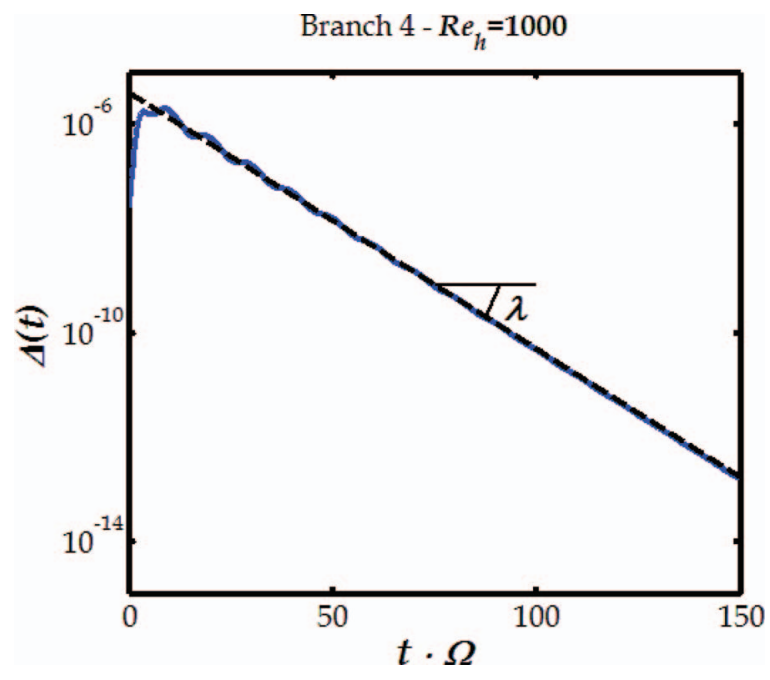

FIG. 3. The Lyapunov coefficient $\lambda$ is determined via a log-linear fit of $\Delta(t)$ for all stable solution branches at $R e_{h}=500$, 750 , and 1000 .

exponentially decay in time? For a Lyapunov-stable solution branch it holds that

$$
\lim _{\substack{t \rightarrow \infty \\ \Delta(0) \rightarrow 0}}\left[\frac{1}{t} \ln \left(\frac{\Delta(t)}{\Delta(0)}\right)\right]=\lambda,
$$

in which the Lyapunov coefficient $\lambda$ is a measure of the stability of the system. For Lyapunov stable solution branches, a plot of $\ln \Delta(t)$ versus $t$ results in a straight line with slope $\lambda<0$ as $t \rightarrow \infty$ (Figure 3 ). The more negative $\lambda$ is, the faster the solution branch converges back to its original state and thus the more stable the solution branch is.

For the second case, in which $\Delta(t) \rightarrow \alpha$ as $t \rightarrow \infty$, the original steady state is not retained and another steady solution branch is found. This new solution branch will represent a new balance of transport of angular momentum, $\Gamma(z, r, t)=G(z, t) r^{2}$. The transition from the original steady state to the new steady state will be characterized according to the mechanism with which the angular momentum is transferred from the original situation to the final situation.

The transport of angular momentum is described by

$$
\frac{\partial \Gamma}{\partial t}+v_{r} \frac{\partial \Gamma}{\partial r}+v_{z} \frac{\partial \Gamma}{\partial z}=\operatorname{Re}_{h}^{-1}\left(\frac{\partial^{2} \Gamma}{\partial z^{2}}+\frac{\partial^{2} \Gamma}{\partial r^{2}}-\frac{1}{r} \frac{\partial \Gamma}{\partial r}\right)
$$

After substituting $\Gamma(z, r, t)=G(z, t) r^{2}$, application of the similarity transformation in Eqs. (10)-(12), and dividing by $r^{2}$, an expression for the transport of angular momentum in terms of the similarity functions $G(z, t)$ and $H(z, t)$ is obtained

$$
\frac{\partial G}{\partial t}=R e_{h}^{-1} \frac{\partial^{2} G}{\partial z^{2}}+2 H \frac{\partial G}{\partial z}-2 G \frac{\partial H}{\partial z} .
$$

Not surprisingly, this equation is the same as the result obtained from the azimuthal component of the Navier-Stokes equations in Eq. (14). However, via Eq. (23), it is now possible to identify the terms leading to a change of the azimuthal velocity to its sources:

- $R e_{h}^{-1} \cdot\left(\partial^{2} G / \partial z^{2}\right)$ represents the diffusive transport of angular momentum in the axial direction,

- $2 H \cdot(\partial G / \partial z)$ describes the convective transport of angular momentum in the axial direction,

- $-2 G \cdot(\partial H / \partial z)$ denotes the convective transport of angular momentum in the radial direction. 


\section{RESULTS}

Section V A starts by presenting and discussing the numerical results of the development of the flow field starting from three situations. A fluid, which is initially at rest $(s=0)$ will be shown to develop into a Batchelor-type of flow field via an intermediate, meta-stable Stewartson-type of flow. When numerical calculations were started with a fluid which was initially in weak counter-rotation with respect to the rotating disk $(s=-0.1)$, a solution branch was obtained, different from both Batchelor's or Stewartson's predictions. This steady type of flow is termed "Branch 3" type flow. Similarly, starting with a fluid in exact counter-rotation with the rotating disk $(s=-1)$ another steady flow field was obtained, which is termed "Branch 4" type of flow. It will be shown that the solutions corresponding to Branch 3, Branch 4, and Batchelor-types of flow are Lyapunovstable. The Stewartson-branch was found to be unstable and the flow field will be shown to develop from Stewartson-type of flow to a Batchelor-type of flow field. Finally, the mechanism behind this transition will be identified as being dominated by radial convection of angular momentum towards the axis of rotation.

\section{A. Variation of initial conditions}

The temporal evolution of the numerical solution in time, starting from a fluid at rest (i.e., $s=0$ ) and $R e_{h}=1000$, is shown in Figure 4. In panel (a), the development of the azimuthal velocity profile is depicted, while panel (b) shows the radial velocity profile. The sequence in time between curves is indicated by an arrow in which the time increment between subsequent curves is equal to $\delta t=t_{f} / 200=25.3$. At $t=0$ the bottom disk is impulsively set in motion, resulting in the very rapid formation of a Stewartson-type of flow. In this flow type, a Von Kármán boundary layer exists near the rotating disk in which the azimuthal velocity of the fluid rapidly decreases from the azimuthal velocity of the disk to zero while moving away from the disk, so that the main part of the fluid is non-rotating. Fluid is also moving radially outwards due to the centrifugal motion of the disk, which is balanced by continuity by a weak axial flow into the Von Kármán boundary layer.

At first, the curves for the velocity profiles are very close together, indicating very small changes in the flow field over time. However, this near-steady solution rapidly changes after about $t=150$. At this point, one observes an increase in azimuthal velocity in the core region of the fluid. The situation then becomes unstable and diverges from a Stewartson-type of flow to a Batchelor-type of flow, in which a Von Kármán boundary layer and a Bödewadt boundary layer are present at the rotating and stationary disk, respectively. The fluid in the core region is behaving as if it were in inviscid, rotational motion. It should be noted that up to this point no disturbance has been added to the system other than small inherent disturbances present in any numerical calculations. It therefore
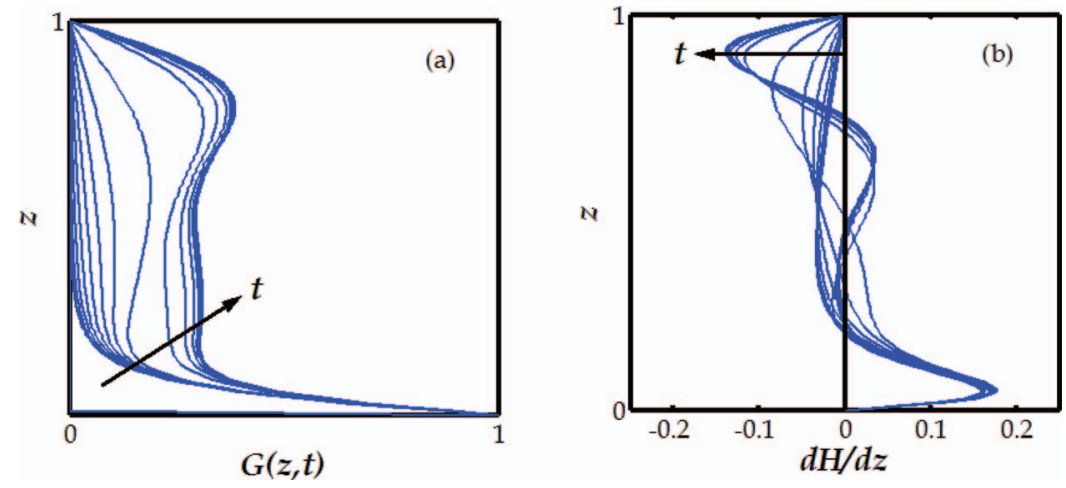

FIG. 4. A fluid which is initially at rest (i.e., $s=0$ ) rapidly develops a flow structure described by Stewartson-type of flow. This intermediate flow field, however, is unstable and evolves into a Batchelor-type of flow. The transition from a Batchelor-type to a Stewartson-type flow occurs around $t=150$. The azimuthal and radial components of the velocity vector during this transition are given in panels (a) and (b), respectively. $\left(R e_{h}=1000, t_{f}=505, \delta t=t_{f} / 200=25.3\right)$. 

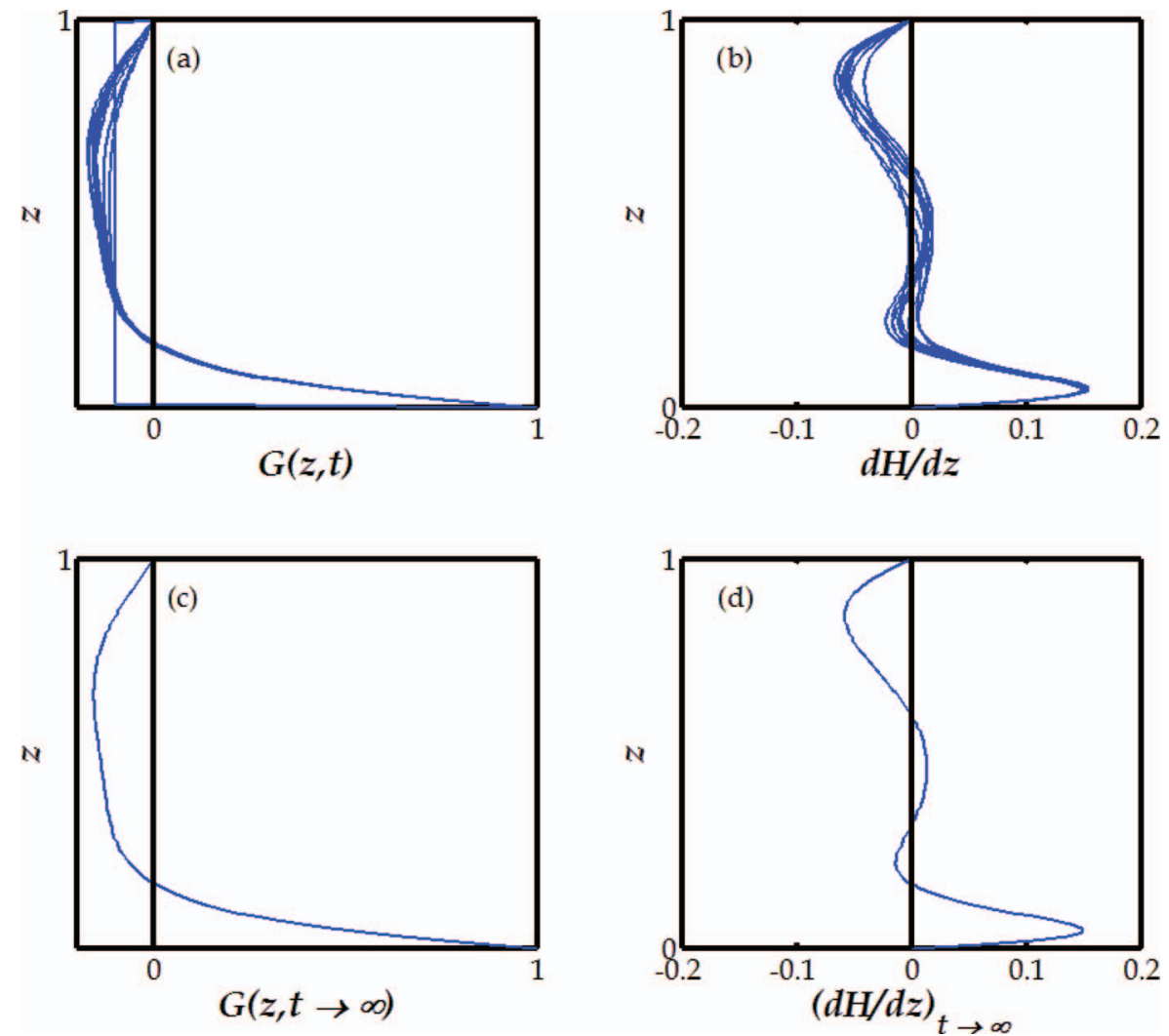

FIG. 5. A fluid which is initially in mild counter-rotation $(s=-0.1)$ with respect to the bottom disk develops a flow structure which retains most of the negative azimuthal velocity, but is very similar to a Batchelor-type of flow. The flow field converges via a damped oscillation towards the steady situation. The oscillatory nature of the flow makes a graphical representation of the temporal evolution of the flow field less clear. For clarity reasons, the steady velocity profiles have been given as well. In panels (a) and (b), the temporal evolution of the azimuthal and radial velocity profiles is depicted, respectively. The ultimate, steady azimuthal, and radial velocity profiles are shown in panels (c) and (d), respectively. $\left(R e_{h}=1000, t_{f}=250\right.$, $\delta t=t_{f} / 200=12.6$ ).

seems that the Stewartson-solution might not be a stable solution of the Navier-Stokes equations for flow between two infinite disks.

In Figure 5, the numerical solution is given starting from a fluid in weak counter-rotation with respect to the rotation of the disk. In this case, the fluid is initially in solid-body counter-rotation with $s=-0.1$, and $R e_{h}=1000$. Panel (a) shows the development of the azimuthal velocity, whereas the development of the radial velocity is given in panel (b). In panels (c) and (d), the azimuthal and radial components of the steady flow field are given for $t=250$, respectively. The fluid velocity profiles can be described as a damped oscillation around the steady flow field, making it difficult to indicate the direction of time between the curves in this figure. This steady type of flow is termed "Branch 3" type of flow. Although the main body of the fluid retains the negative azimuthal velocity, the flow structure is very similar to a Batchelor-type of flow, in which both a Von Kármán and a Bödewadt boundary layer are separated by a bulk region of uniform azimuthal velocity.

The numerical solution, starting from a fluid in counter-rotation with respect to the rotation of the disk, is depicted in Figure 6. In this situation, the fluid is initially in solid-body counter-rotation with $s=-1$, and $R e_{h}=1000$. The development of the azimuthal and radial components of the velocity vector is given in panels (a) and (b), respectively. The time increment between subsequent curves is equal to $\delta t=t_{f} / 200=1.26$. At $t=0$, the bottom disk is impulsively set in motion, resulting in the very rapid formation of a type of flow, which is termed "Branch 4" type of flow. The flow field again retains most of its negative azimuthal velocity, but the flow field is very different from either Branch 3, Batchelor or Stewartson-types of flow. There is no clear distinction between a boundary 

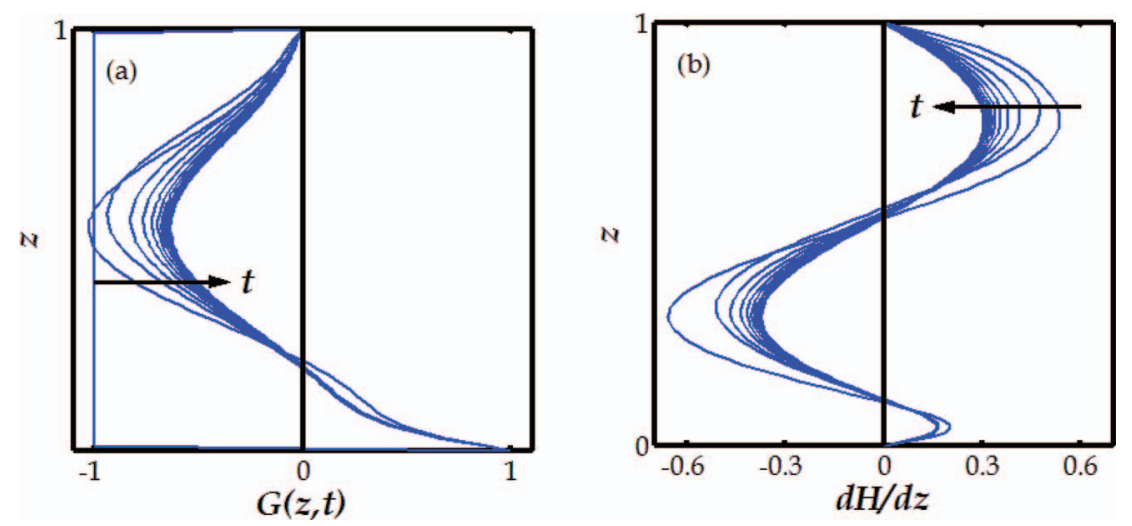

FIG. 6. A fluid which is initially in counter-rotation $(s=-1)$ with respect to the bottom disk develops a flow structure which retains most of the negative azimuthal velocity. Panels (a) and (b) show the azimuthal and radial velocity components, respectively. $\left(R e_{h}=1000, t_{f}=251.3, \delta t=t_{f} / 200=1.26\right)$.

layer and a center region of the flow field, as the changes in velocity over the axial coordinate are more gradual and spread out over the entire region between the disks. Also, one observes radial outflow at both the rotating and the stationary disk.

\section{B. Stability analysis}

Starting with the steady velocity profiles for Branch 3, Branch 4, and Batchelor-flow, a random perturbation is added at $t=0$. The disturbance propagation in time is monitored by means of the function $\Delta(t)$, as defined in Eq. (20). For each of the three stable solutions a stability analysis was performed at $R e_{h}=500,750$, and 1000. This stability analysis consists of a set of 20 simulations per branch for each selected value of the Reynolds number. The resulting data for $\Delta(t)$ were then fitted to Eq. (21) on a log-linear scale to obtain the Lyapunov-coefficient. A Discrete Fourier Analysis was performed on the data to determine the frequencies of the oscillations that were present in the data for $\Delta(t)$.

The Lyapunov-coefficients for the Batchelor, Branch 3 and Branch 4 solutions are depicted in Figure 7. The Lyapunov-coefficients all have a negative value, confirming their stability to a minor disturbance. The Lyapunov-coefficients of Branch 4 type of flow are lower than those for Batchelor-type of flow, which are again lower than those for Branch 3 type of flow. This indicates a trend in relative stability, in which Branch 4 types of flow are very stable to a minor disturbance, in the sense that they return more rapidly to the original flow field than the Branch 3 type of flow. From Figure 8(a), it can again be seen that Branch 3 hosts an oscillation with a characteristic frequency $f$. From a Discrete Fourier Analysis on the data of $\Delta(t)$ for Branch 3 at $R e_{h}=500,750$, and 1000, it was observed that the frequency of the oscillations increases with the Reynolds number, as shown in Figure 8(b). Also note that the amplitude of the frequency appears to be constant over time in the logarithmic scale of Figure 8(a), but this is a visual artefact from the logarithmic scaling of the vertical axis. In fact, the amplitude of the oscillation is decreasing exponentially as well.

\section{Instability of the Stewartson solution}

Under the Von Kármán similarity hypothesis, Stewartson-type of flow seems to be an unstable solution of the steady Navier-Stokes equations for rotationally symmetric flow between two disks. It appears to be an intermediate flow field in the development of the flow from a fluid at rest to a Batchelor-type of flow. From the evolution equation (22) of angular momentum, it is possible to track the change of angular momentum in terms of radial, axial, diffusive, and convective transport during the transition from Stewartson to Batchelor-type of flow, as depicted in Figure 9. 


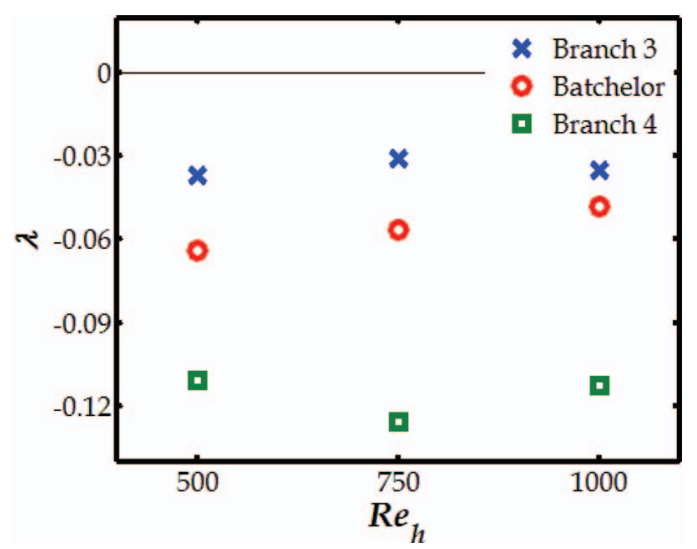

FIG. 7. The Lyapunov coefficients for the three stable solution branches. Branch 4 is more stable than the Batchelor branch, while Branch 3 is the least stable of these solutions.
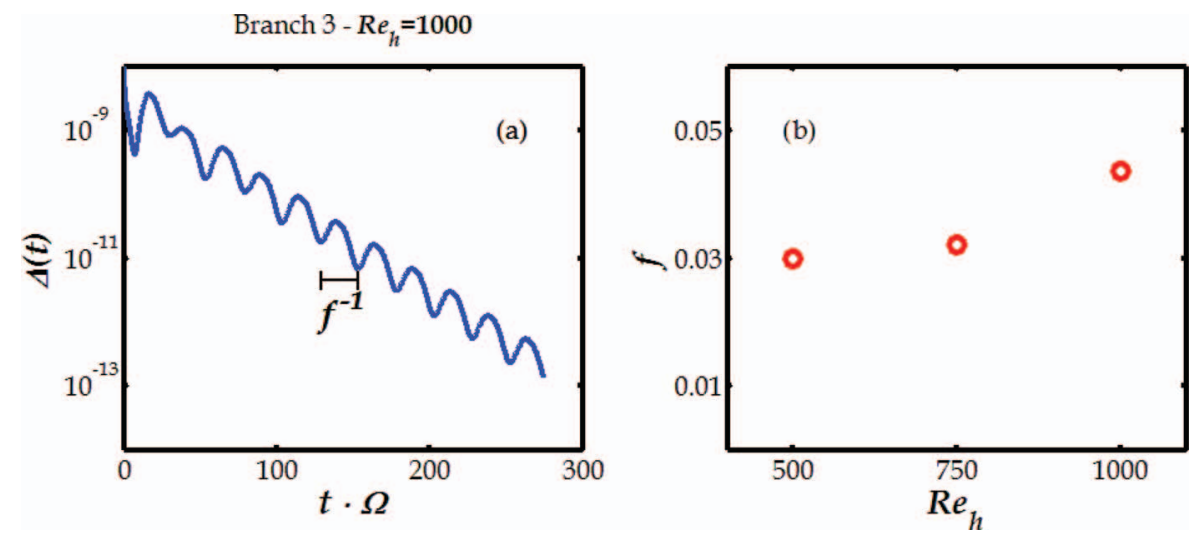

FIG. 8. (a) Branch 3 hosts an oscillation in the flow field which amplitude exponentially decays in line with the decay of the magnitude of the disturbance propagation itself. (b) The frequency of oscillation $f$ as a function of the Reynolds number $R e_{h}$ is determined via a Discrete Fourier transform.
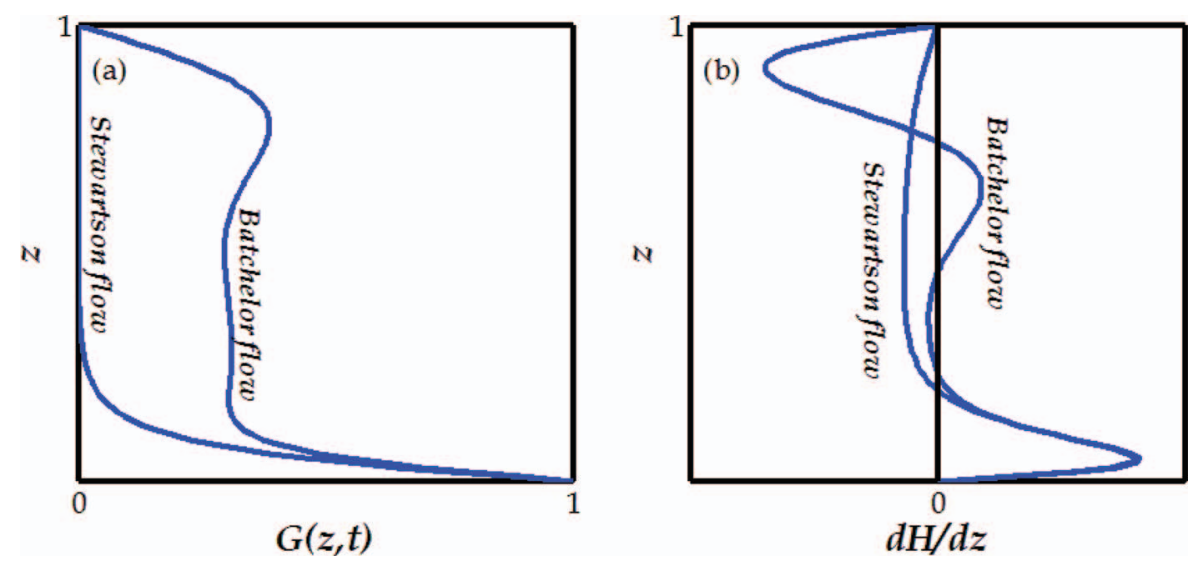

FIG. 9. During the transition from Stewartson to Batchelor-flow, the (a) azimuthal velocity increases from near-zero to $0.313 \Omega r$, in line with earlier findings by Dijkstra and Van Heijst (1983), ${ }^{9}$ whereas the (b) radial velocity profile develops a Bödewadt boundary layer. 

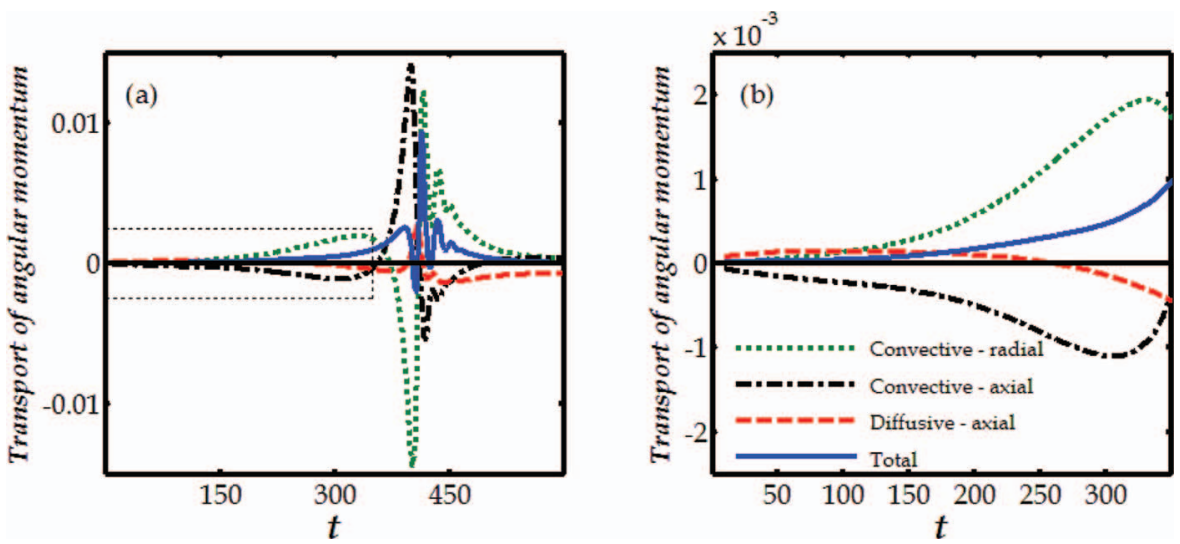

FIG. 10. (a) The dimensionless magnitude of the different transport terms for the transport of angular momentum are plotted versus time during the transition from a fluid at rest, via a Stewartson-type of flow, to a Batchelor-type of flow. In panel (b), only the period of time is considered in which a fluid at rest develops into an unstable intermediate type of flow.

Figure 10 presents graphs of the transport of angular momentum associated with convective transport in the radial and axial direction and diffusive transport in the axial direction. Figure 10(a) shows the entire time frame from a fluid at rest to fully developed Batchelor-flow, while Figure 10(b) only shows a limited time interval, during which the change from Stewartson-flow to an unstable intermediate type of flow takes place. It can clearly be seen that the process becomes unstable as soon as the radial transport of angular momentum by convection becomes dominating, triggering an unstable flow field which changes from a Stewartson-type flow to a Batchelor-type solution. This can be understood by realizing that the angular momentum in the core region, however small, is not exactly zero and this non-zero angular momentum is convectively transported from larger radii to smaller ones. Then, by conservation of angular momentum, the azimuthal velocity increases, leading to an azimuthal acceleration of the fluid in the core region.

It should be noted that Stewartson-types of flow have been observed experimentally ${ }^{10}$ where a finite rotating disk is moving near a stationary disk without an encasing cylindrical wall; i.e., the fluid between the edges of the disks is unbounded. Stewartson-flow is also observed in systems where there is a superimposed centrifugal outflow between the disks. ${ }^{11}$ In an unbounded configuration, fresh incoming fluid with negligible angular momentum is constantly replacing the rotational outgoing fluid in the Von Kármán boundary layer. For the case of a net radial outflow, the outflowing fluid acts as a sink for the angular momentum by convectively transporting it out of the region between the disks. It might be that these two mechanisms of transport of angular momentum away from the axis of rotation hamper the buildup of azimuthal velocity in the center region between the disks and thus prevent the trigger of an instability leading to Batchelor-flow.

In fact, when a cylindrical housing was set to co-rotate with the rotating disk and radial transport of angular momentum towards the axis of rotation was thus not as strongly limited, Batchelor-types of flow were indeed found both experimentally ${ }^{9}$ and numerically. $8,9,12,13$

\section{CONCLUDING REMARKS}

The time-dependent Navier-Stokes equations for the rotationally symmetric flow between two infinite disks are numerically solved, under the Von Kármán similarity assumption, using an implicit finite difference method. Three different steady solution branches were found as a result of varying the initial condition of the fluid:

- A fluid initially at rest develops via a Stewartson-type flow field to a Batchelor-type of flow.

- A fluid which is initially in weak counter-rotation with respect to the motion of the rotating disk (i.e., $\left.\underline{\hat{v}}_{0}=(0,-0.1 \Omega \hat{r}, 0)\right)$ evolves into a type of flow (Branch 3$)$ which retains most of its negative azimuthal velocity. The flow field is very similar to a Batchelor-type of flow, 
consisting of Von Kármán and Bödewadt boundary layers at the disks, separated by a region of uniform (but negative) azimuthal velocity.

- A fluid which is initially in complete counter-rotation with respect to the motion of the rotating disk (i.e., $\underline{\hat{v}}_{0}=(0,-\Omega \hat{r}, 0)$ ) evolves into a type of flow (Branch 4$)$ which also retains most of its negative azimuthal velocity. However, the flow field does not allow for a definition of different regions in the fluid, since the velocity gradients are more gradually spread out over the entire region between both disks in comparison to the other types of flow.

Furthermore, it was found that three of these solution branches were stable to a minor disturbance and the propagation of this disturbance can be described by Lyapunov stability theory. Branch 4 was found to be the most stable solution, with the lowest Lyapunov-coefficient $\lambda$. The Batchelor-solution was stable to a minor disturbance as well, but its Lyapunov-coefficient was higher. The least stable of the stable solution branches was found to be Branch 3. Not only did this branch have the smallest negative $\lambda$-value, the system also harbored a characteristic oscillation with a frequency that increases with an increasing Reynolds number.

The unstable solution branch, the Stewartson-type of flow, already diverged from its initial state by disturbances that are inherently present in numerical calculations. The flow field develops from a Stewartson-type of flow in which a Bödewadt boundary layer and a core region with uniform azimuthal velocity are absent, to a Batchelor-type of flow. The process with which the core region obtains its azimuthal velocity was found to be triggered and dominated by the convective transport of angular momentum from larger to smaller radii.

The experimental procedure of adding a random disturbance conforming to the Von Kármán similarity hypothesis might not be physically feasible. However, the theoretical discussions on the existence and multiplicity of solutions for rotating disk flows in the 20th century also were held under this similarity hypothesis. In that light, it thus appears that Stewartson's claim on the structure of the steady flow field between two infinite rotating disks might be incorrect and that the Stewartson-type of flow was actually an intermediate, meta-stable flow field in the evolution of a fluid from rest to a flow structure more reminiscent of Batchelor-flow.

The presence of a cylindrical wall at a finite radial distance will have an important effect on the stability of the flow field in the center region of the fluid as this end region will have an additional stabilizing or destabilizing effect on the radial transport of angular momentum. In finite systems, in which the fluid near the edge of the disks is unbounded, or where there is a superimposed centrifugal outflow, the radial transport of angular momentum towards the axis of rotation will be limited, preventing the trigger of an instability in the Stewartson-type flow field. This might explain the physical existence of Stewartson-types of flow in open rotor-stator systems of finite dimensions or systems in which there is a superimposed centrifugal outflow. Although a Batchelortype of flow would be expected from the stability of the self-similar solution branches, the build-up of azimuthal velocity and a non-viscous core is hindered, because the limited radial transport of angular momentum towards the axis of rotation prevents the triggering of the instability.

\section{ACKNOWLEDGMENTS}

The authors gratefully acknowledge the European Research Council (ERC) for their financial support to this project (ERC Contract No. 227010).

${ }^{1}$ T. von Kármán, “Über laminare und turbulente Reibung,” ZAMM 1, 233-252 (1921).

${ }^{2}$ K. M. P. van Eeten, J. van der Schaaf, J. C. Schouten, and G. J. F. van Heijst, "Boundary layer development in the flow field between a rotating and a stationary disk," Phys. Fluids 24, 033601 (2012).

${ }^{3}$ U. T. Bödewadt, "Die Drehströmung über festem Grunde," ZAMM 20, 241-253 (1940).

${ }^{4}$ G. K. Batchelor, "Note on a class of solutions of the Navier-Stokes equations representing steady rotationally-symmetric flow," Q. J. Mech. Appl. Math. 4, 29-41 (1951).

${ }^{5}$ K. Stewartson, "On the flow between two rotating coaxial disks," Math. Proc. Cambridge Philos. Soc. 49, 333-341 (1953).

${ }^{6}$ M. Holodniok, M. Kubíček, and V. Hlaváček, "Computation of the flow between two rotating coaxial disks," J. Fluid Mech. 81, 689-699 (1977).

${ }^{7}$ M. Holodniok, M. Kubíček, and V. Hlaváček, "Computation of the flow between two rotating coaxial disks: Multiplicity of steady solutions," J. Fluid Mech. 108, 227-240 (1981). 
${ }^{8}$ J. M. Lopez, F. M. Marques, A. M. Rubio, and M. Avila, "Crossflow instability of finite Bödewadt flows: Transients and spiral waves," Phys. Fluids 21, 114107 (2009).

${ }^{9}$ D. Dijkstra and G. J. F. van Heijst, "The flow between two finite rotating disks enclosed by a cylinder," J. Fluid Mech. 128, 123-154 (1983).

${ }^{10}$ M. Djaoui, A. Malesys, and R. Debuchy, "On the experimental evidence of the sensitiveness of rotor-stator flows with regard to peripheral boundary conditions," C. R. Acad. Sci., Ser. IIb Mec. 327, 49-54 (1999).

${ }^{11}$ S. Poncet, M. P. Chauve, and R. Schiestel, "Batchelor versus Stewartson flow structures in a rotor-stator cavity with throughflow," Phys. Fluids 17, 075110 (2005).

${ }^{12}$ H. P. Pao, “A numerical computation of a confined rotating flow," J. Appl. Mech. 37, 480-487 (1970).

${ }^{13}$ J. M. Lopez, "Characteristics of endwall and sidewall boundary layers in a rotating cylinder with a differentially rotating endwall," J. Fluid Mech. 359, 49-79 (1998). 